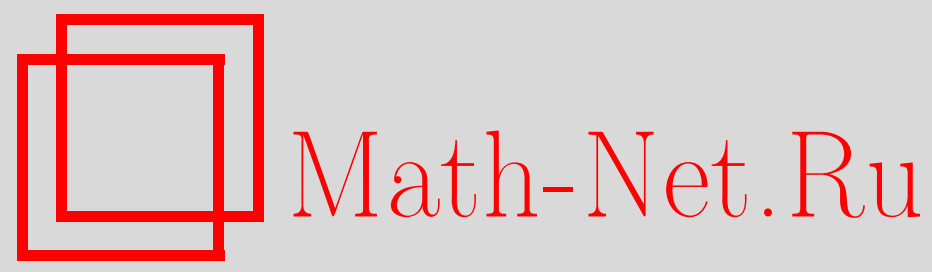

С. В. Смышляев, Барьеры совершенно уравновешенных булевых функций, Дискрет. матем., 2010, том 22, выпуск 2, 66-79

DOI: https://doi.org/10.4213/dm1096

Использование Общероссийского математического портала Math-Net.Ru подразумевает, что вы прочитали и согласны с пользовательским соглашением http://www.mathnet.ru/rus/agreement

Параметры загрузки:

IP: 35.173 .219 .12

26 апреля 2023 г., 14:15:09 


\title{
Барьеры совершенно уравновешенных булевых функций
}

\author{
(c) 2010 г. С. В. Смышляев
}

\begin{abstract}
В работе продолжено начатое ранее изучение свойств совершенно уравновешенных булевых функций с использованием аппарата барьеров булевых функций.

Работа выполнена при поддержке Российского фонда фундаментальных исследований, проект 09-01-00653-а.
\end{abstract}

\section{1. Введение}

В работе продолжено начатое в [1] изучение свойств совершенно уравновешенных булевых функций с использованием аппарата барьеров булевых функций. Первичный анализ функций с барьером конечной длины позволил выделить их в представляющий особый интерес подкласс совершенно уравновешенных функций, однако также показал необходимость глубокого изучения их свойств, как для анализа слабостей фильтрующих генераторов с обладающими барьером функциями, так и для построения классов функций с барьером конечной длины и классов функций без барьера. В настоящей работе приведено исследование функций с барьером с этих позиций и получены результаты, из которых понятна принципиальная важность знания длины барьера функции при использовании ее в качестве фильтрующей. Кроме того, получен результат о длинах барьеров функций, построенных с помощью предложенной в работе [2] конструкции, а также приведены некоторые следствия из него, важные для построения определенных классов совершенно уравновешенных функций.

В разделе 2 приводятся основные используемые в работе определения и обозначения, в разделе 3 - необходимые для изложения предварительные результаты. В разделе 4 приводятся формулировки и доказательства основных результатов: доказывается утверждение, описывающее некоторые криптографические слабости булевых функций с конечным барьером, рассматривается один из возможных подходов к построению широких классов совершенно уравновешенных функций с большой длиной барьера и без барьера, приводится конкретный метод построения функций без барьера, позволяющий строить такие функции, существенно зависящие от сколь угодно большого числа переменных, а также доказываются некоторые другие свойства функций с барьером. В разделе 5 намечены некоторые направления дальнейших исследований. 


\section{2. Основные определения и обозначения}

Будем использовать следующие обозначения: $F_{2}$ - поле Галуа из двух элементов, $V_{n}=F_{2}^{n}$ - пространство наборов длины $n \in \mathbf{N}$ над полем $F_{2}$. Будем обозначать через $\mathscr{F}_{n}$ множество булевых функций от $n$ переменных $\left\{x_{1}, x_{2}, \ldots, x_{n}\right\}$. Крайними переменными функции $f \in \mathscr{F}_{n}$ будем называть $x_{1}$ и $x_{n}$. Пусть $m \in \mathbf{N}$. Рассмотрим систему булевых уравнений

$$
f\left(x_{s}, x_{s+1}, \ldots, x_{s+n-1}\right)=y_{s}, \quad s=1,2, \ldots, m .
$$

Система (1) описывает функционирование (в смысле зависимости выходных символов от входных) в течение $m$ тактов кодирующего устройства (см. [3]), построенного с помощью регистра сдвига с $n$ ячейками двоичной памяти и булевой функции $f$ как фильтрующей функции.

Для любой $f \in \mathscr{F}_{n}$ через $f^{*}$ обозначим отображение из $V_{m+n-1}$ в $V_{m}$ следующего вида:

$$
f^{*}\left(x_{1}, x_{2}, \ldots, x_{m+n-1}\right)=\left(f\left(x_{1}, \ldots, x_{n}\right), f\left(x_{2}, \ldots, x_{n+1}\right), \ldots, f\left(x_{m}, \ldots, x_{m+n-1}\right)\right) .
$$

Введем понятие дефекта булевой функции, определив для любой $f \in \mathscr{F}_{n}$ и произвольного $m \in \mathbf{N}$ множество

$$
J(f, m)=V_{m} \backslash \operatorname{Im}\left(f^{*}\right)
$$

и величину

$$
\operatorname{Def}_{m}(f)=|J(f, m)|,
$$

где $|M|$ - мощность множества $M$. В работе [4] элементы множества $J(f, m)$ называются запретами функции $f$ длины $m$.

Определение 1 ([4]). Булева функция $f \in \mathscr{F}_{n}$ называется функцией дефекта нуль, если $\operatorname{Def}_{m}(f)=0$ для любого $m \in \mathbf{N}$.

Замечание 1. Нетрудно заметить (см. [4]), что если функция линейна хотя бы по одной из крайних переменных, то она является функцией дефекта нуль. Обозначим множество функций из $\mathscr{F}_{n}$, линейных по первой переменной, через $\mathscr{L}_{n}$, а множество функций из $\mathscr{F}_{n}$, линейных по последней переменной, через $\mathscr{R}_{n}$.

Определение 2 ([4]). Булева функция $f \in \mathscr{F}_{n}$ называется совершенно уравновешенной, если соотношение

$$
\left|\left(f^{*}\right)^{-1}(y)\right|=2^{n-1}
$$

выполняется для любого $m \in \mathbf{N}$ и любого $y \in V_{m}$.

\section{3. Предварительные результаты}

Теорема 1 ([4]). Булева функция $f \in \mathscr{F}_{n}$ является функцией дефекта нуль тогда и только тогда, когда она совершенно уравновешена.

Множество совершенно уравновешенных функций (функций дефекта нуль) из $\mathscr{F}_{n}$ обозначим $\mathscr{P g}_{n}$. 
Следующие преобразования множества $\mathscr{F}_{n}$ оставляют инвариантным множество $\mathscr{P}_{S_{n}}$ (см. [4]):

$\left(1^{\circ}\right) \gamma_{0}: f\left(x_{1}, \ldots, x_{n}\right) \rightarrow f\left(x_{1}, \ldots, x_{n}\right) \oplus 1$;

$\left(2^{\circ}\right) \gamma_{1}: f\left(x_{1}, \ldots, x_{n}\right) \rightarrow f\left(x_{1} \oplus 1, \ldots, x_{n} \oplus 1\right)$

$\left(3^{\circ}\right) \gamma_{2}: f\left(x_{1}, \ldots, x_{n}\right) \rightarrow f\left(x_{n}, \ldots, x_{1}\right)$.

Особая роль класса совершенно уравновешенных функций как фильтрующих видна из условий равномерности распределения правых частей системы уравнений (1) при условии равномерности распределения вектора $X_{m}=\left(x_{1}, \ldots, x_{m+n-1}\right)$.

Теорема 2 ([5]). Пусть $n \in \mathbf{N} u f \in \mathscr{F}_{n}$. Пусть $\left\{X_{m}=\left(x_{1}, \ldots, x_{m+n-1}\right)\right\}_{m=1}^{\infty}-$ последовательность случайных векторов с распределением

$$
\mathbf{P}\left\{X_{m}=\left(a_{1}, \ldots, a_{m+n-1}\right)\right\}=2^{-(m+n-1)}
$$

для любых $\left(a_{1}, \ldots, a_{m+n-1}\right) \in V_{m+n-1}$. Случайный вектор $Y_{m}=f^{*}\left(X_{m}\right)$ распределен равномерно для любого $m \in \mathbf{N}$ тогда и только тогда, когда $f$-совершенно уравновешенная функция.

Теорема 3 ([4]). Булева функция $f \in \mathscr{F}_{n}$ является совершенно уравновешенной тогда $u$ только тогда, когда не существует двух двоичных последовательностей

$$
x=\left(x_{1}, \ldots, x_{r}\right), \quad z=\left(z_{1}, \ldots, z_{r}\right) \in V_{r}, \quad r>2 n,
$$

таких, что

$$
\begin{aligned}
x_{1} & =z_{1}, \ldots, x_{n}=z_{n}, \quad x_{r-n+1}=z_{r-n+1}, \ldots, x_{r}=z_{r} ; \\
x & \neq z ; \\
f\left(x_{i}, \ldots, x_{i+n-1}\right) & =f\left(z_{i}, \ldots, z_{i+n-1}\right), \quad i=1, \ldots, r-n+1 .
\end{aligned}
$$

Для пары натуральных чисел $m, k$ рассмотрим отображение $\Xi_{m, k}$ из $\mathscr{F}_{m} \times \mathscr{F}_{k}$ в $\mathscr{F}_{m+k-1}$ вида

$$
\Xi_{m, k}(g, h)=g[h]=f \in \mathscr{F}_{m+k-1}, \quad g \in \mathscr{F}_{m}, \quad h \in \mathscr{F}_{k},
$$

где

$$
\begin{aligned}
f\left(x_{1}, \ldots, x_{m+k-1}\right) & =g[h]\left(x_{1}, \ldots, x_{m+k-1}\right) \\
& =g\left(h\left(x_{1}, \ldots, x_{k}\right), h\left(x_{2}, \ldots, x_{k+1}\right), \ldots, h\left(x_{m}, \ldots, x_{m+k-1}\right)\right) .
\end{aligned}
$$

Для этой конструкции справедливо следующее утверждение.

Теорема 4 ([2]). Пусть $g \in \mathscr{F}_{m}, h \in \mathscr{F}_{k}$. Функция $f=g[h] \in \mathscr{F}_{m+k-1}$ совершенно уравновешена тогда и только тогда, когда функции $g$ и $h$ совершенно уравновешены.

Утверждение теоремы 4 позволяет строить булевы функции из $\mathscr{P}_{S_{n}}$, не входящие в классы $\mathscr{L}_{n}$ и $\mathscr{R}_{n}$. 
Пример 1 ([2]). Пусть

$$
\begin{aligned}
& g\left(x_{1}, x_{2}, x_{3}\right)=x_{1} \oplus x_{2} x_{3} \in \mathscr{L}_{3}, \\
& h\left(x_{1}, x_{2}, x_{3}\right)=x_{1} x_{2} \oplus x_{3} \in \mathscr{R}_{3} .
\end{aligned}
$$

Тогда функция

$$
\begin{aligned}
f\left(x_{1}, x_{2}, x_{3}, x_{4}, x_{5}\right) & =g\left(h\left(x_{1}, x_{2}, x_{3}\right), h\left(x_{2}, x_{3}, x_{4}\right), h\left(x_{3}, x_{4}, x_{5}\right)\right) \\
& =x_{1} x_{2} \oplus x_{3} \oplus x_{2} x_{3} x_{4} \oplus x_{2} x_{3} x_{5} \oplus x_{3} x_{4} \oplus x_{4} x_{5} \notin \mathscr{L}_{5} \cup \mathscr{R}_{5}
\end{aligned}
$$

является совершенно уравновешенной.

Определение 3 ([1]). Булева функция $f \in \mathscr{F}_{n}$ называется функцией с правым барьером длины $b$, если система уравнений

$$
\begin{aligned}
f\left(y_{1}, y_{2}, \ldots, y_{n}\right) & =f\left(z_{1}, z_{2}, \ldots, z_{n}\right) \\
f\left(y_{2}, y_{3}, \ldots, y_{n+1}\right) & =f\left(z_{2}, z_{3}, \ldots, z_{n+1}\right) \\
& \ldots \\
f\left(y_{b-1}, y_{b}, \ldots, y_{b+n-2}\right) & =f\left(z_{b-1}, z_{b}, \ldots, z_{b+n-2}\right) \\
y_{1}=z_{1}=x_{1}, \ldots, y_{n-1} & =z_{n-1}=x_{n-1}, y_{n}=0, z_{n}=1
\end{aligned}
$$

имеет решение, а система

$$
\begin{aligned}
f\left(y_{1}, y_{2}, \ldots, y_{n}\right) & =f\left(z_{1}, z_{2}, \ldots, z_{n}\right) \\
f\left(y_{2}, y_{3}, \ldots, y_{n+1}\right) & =f\left(z_{2}, z_{3}, \ldots, z_{n+1}\right) \\
& \ldots \\
f\left(y_{b-1}, y_{b}, \ldots, y_{b+n-2}\right) & =f\left(z_{b-1}, z_{b}, \ldots, z_{b+n-2}\right) \\
f\left(y_{b}, y_{b+1}, \ldots, y_{b+n-1}\right) & =f\left(z_{b}, z_{b+1}, \ldots, z_{b+n-1}\right) \\
y_{1}=z_{1}=x_{1}, \ldots, y_{n-1} & =z_{n-1}=x_{n-1}, y_{n}=0, z_{n}=1
\end{aligned}
$$

решений не имеет.

Булева функция $f \in \mathscr{F}_{n}$ называется функцией с левым барьером длины $b$, если $f^{\gamma_{2}}\left(x_{1}, \ldots, x_{n}\right) \equiv f\left(x_{n}, \ldots, x_{1}\right)$ является функцией с правым барьером длины $b$.

Булева функция $f \in \mathscr{F}_{n}$ имеет барьер, если она имеет правый или левый барьер, или оба сразу. При этом длиной барьера функции называется соответственно длина правого барьера, левого барьера, или меньшая из длин барьеров.

Наличие барьера у функции является достаточным, но не необходимым условием совершенной уравновешенности функции. Длина барьера - величина, определенным образом характеризующая совершенно уравновешенную функцию. Из представленных ниже результатов понятно, что в множестве $\mathscr{P} \mathscr{S}_{n}$ наибольший интерес представляют функции с большой длиной барьера, а также функции без барьера.

Теорема 5 ([1]). Пусть $f \in \mathscr{F}_{n}-$ функиия с правым (левым) барьером длинь $b, b<n, a$ $g \in \mathscr{F}_{n-b}-$ произвольная функция. Тогда функция

$$
h\left(x_{1}, \ldots, x_{n}\right)=f\left(x_{1}, \ldots, x_{n}\right) \oplus g\left(x_{1}, \ldots, x_{n-b}\right)
$$

(соответственно, $\left.h\left(x_{1}, \ldots, x_{n}\right)=f\left(x_{1}, \ldots, x_{n}\right) \oplus g\left(x_{b+1} \ldots, x_{n}\right)\right)$ тоже является функциией с правым (левым) барьером длины $b$. 
В теореме 4 доказано, что отображение $\Xi_{m, k}(g, h)$ сохраняет свойство совершенной уравновешенности функций $g$ и $h$, что делает возможным построение совершенно уравновешенных функций от большого числа переменных с помощью известных совершенно уравновешенных функций. Интересно рассмотреть вопрос о барьерах полученной функции $f=\Xi_{m, k}(g, h)$, чтобы иметь возможность, например, строить совершенно уравновешенные функции от любого числа переменных, не имеющие барьеров, являющиеся наиболее предпочтительными для использования в качестве фильтрующих функций.

\section{4. Основные результаты}

Лемма 1. Если $f \in \mathscr{P P B}_{n}$, то для любого натурального $u$, для любых наборов $z_{0}, z_{1} \in V_{u}$ существуют натуральное число $r$ и наборы $x, y \in V_{r+n-1}, z \in V_{r-u}$ такие, что выполнена система

$$
\begin{gathered}
x_{1}=y_{1} \\
\ldots \\
x_{n-1}=y_{n-1} \\
f^{*}(x)=\left(z \mid z_{0}\right) \\
f^{*}(y)=\left(z \mid z_{1}\right)
\end{gathered}
$$

Доказательство. Введем для любого $p \in N$ и любого набора $\left(\delta_{1}, \ldots, \delta_{p}\right)$ множество

$$
A_{\left(\delta_{1}, \ldots, \delta_{p}\right)}=\left\{\left(x_{1}, \ldots, x_{n-1}\right) \mid \exists x_{n}, \ldots, x_{p+n-1}: f^{*}\left(x_{1}, \ldots, x_{p+n-1}\right)=\left(\delta_{1}, \ldots, \delta_{p}\right)\right\} .
$$

Тогда условие леммы можно переписать в виде

$$
f \in \mathscr{P M}_{n} \Longrightarrow \forall u \in \mathbf{N}, z_{0}, z_{1} \in V_{u} \Longrightarrow \exists r \in \mathbf{N}, z \in V_{r-u}: A_{\left(z \mid z_{0}\right)} \cap A_{\left(z \mid z_{1}\right)} \neq \varnothing \text {. }
$$

Заметим, что для любых $\left(\delta_{1}, \ldots, \delta_{p}, \delta_{p+1}, \ldots, \delta_{p+k}\right)$ верно соотношение

$$
A_{\left(\delta_{1}, \ldots, \delta_{p+k}\right)} \subseteq A_{\left(\delta_{1}, \ldots, \delta_{p}\right)},
$$

откуда следует, что

$$
\left|A_{\left(\delta_{1}, \ldots, \delta_{p+k}\right)}\right| \leqslant\left|A_{\left(\delta_{1}, \ldots, \delta_{p}\right)}\right|
$$

Из $f \in \mathscr{P}_{n}$ следует, что для любого $k$ и любых $\left(\delta_{1}, \ldots, \delta_{p}, \delta_{p+1}, \ldots, \delta_{p+k}\right)$ верно неравенство $\left|A_{\left(\delta_{1}, \ldots, \delta_{p+k}\right)}\right| \geqslant 1$, тогда существует такое натуральное $C$, что для любого $k$ и любых $\left(\delta_{1}, \ldots, \delta_{p}, \delta_{p+1}, \ldots, \delta_{p+k}\right)$ верно неравенство $\left|A_{\left(\delta_{1}, \ldots, \delta_{p+k}\right)}\right| \geqslant C$ и существует $p^{*} \in \mathbf{N}$ и набор $\left(\delta_{1}^{*}, \ldots, \delta_{p^{*}}^{*}\right) \in V_{p^{*}}:\left|A_{\left(\delta_{1}^{*}, \ldots, \delta_{p^{*}}^{*}\right)}\right|=C$.

Фиксируем произвольное $u \in \mathbf{N}$ и $z_{0}, z_{1} \in V_{u}$. Положим $r=p^{*}+u, z=\left(\delta_{1}^{*}, \ldots, \delta_{p^{*}}^{*}\right)$. Выше доказано, что $A_{\left(z \mid z_{0}\right)} \subseteq A_{(z)}, A_{\left(z \mid z_{1}\right)} \subseteq A_{(z)}$,

$$
\left|A_{\left(z \mid z_{0}\right)}\right|=\left|A_{\left(z \mid z_{1}\right)}\right|=\left|A_{(z)}\right|=C>0,
$$

откуда следует, что $A_{\left(z \mid z_{0}\right)}=A_{\left(z \mid z_{1}\right)}=A_{(z)} \neq \varnothing$. Получаем требуемое соотношение $A_{\left(z \mid z_{0}\right)} \cap A_{\left(z \mid z_{1}\right)} \neq \varnothing$. Лемма доказана. 
Теперь мы можем доказать утверждение, отвечающее на вопрос о барьерах полученных с помощью $\Xi_{m, k}(g, h)$ функций. Далее будем считать длину правого (левого) барьера функции бесконечно большой, если функция не имеет правого (левого) барьера. Докажем утверждение для правых барьеров, для левых барьеров доказательство проводится совершенно аналогично.

Теорема 6. Пусть $h \in \mathscr{F}_{k}, g \in \mathscr{F}_{m}, f=g[h]$, а длины правых барьеров функиий $h, g, f$ равны соответственно $b, c, d$. Тогда выполнено соотношение

$$
\max \{b, c\} \leqslant d \leqslant b+c-1,
$$

где оба неравенства могут обращаться, а могут и не обращаться в равенства.

Доказательство. Сначала рассмотрим случай конечных барьеров $b<\infty, c<\infty$. Тогда, по определению правого барьера длины $b$, система

$$
\begin{aligned}
h\left(y_{m}, y_{m+1}, \ldots, y_{m+k-1}\right) & =h\left(z_{m}, z_{m+1}, \ldots, z_{m+k-1}\right) \\
h\left(y_{m+1}, y_{m+2}, \ldots, y_{m+k}\right) & =h\left(z_{m+1}, z_{m+2}, \ldots, z_{m+k}\right) \\
& \ldots \\
h\left(y_{m+b-1}, y_{m+b}, \ldots, y_{m+b+k-2}\right) & =h\left(z_{m+b-1}, z_{m+b}, \ldots, z_{m+b+k-2}\right) \\
y_{m}=z_{m}=x_{m}, \ldots, y_{m+k-2}=z_{m+k-2} & =x_{m+k-2}, y_{m+k-1}=0, z_{m+k-1}=1
\end{aligned}
$$

не выполнена ни для каких $x_{m}, \ldots, x_{m+k-2}, y_{m+k}, \ldots, y_{m+b+k-2}, z_{m+k}, \ldots, z_{m+b+k-2}$, где $y_{m}=z_{m}=x_{m}, \ldots, y_{m+k-2}=z_{m+k-2}=x_{m+k-2}, y_{m+k-1}=0, z_{m+k-1}=1$. Введем обозначения

$$
\begin{aligned}
& h_{j, 0}=h\left(y_{j}, y_{j+1}, \ldots, y_{j+k-1}\right), \\
& h_{j, 1}=h\left(z_{j}, z_{j+1}, \ldots, z_{j+k-1}\right) .
\end{aligned}
$$

Тогда для любого фиксированного набора $x_{m}, \ldots, x_{m+k-2}, y_{m+k}, \ldots, y_{m+b+k-2}, z_{m+k}$, $\ldots, z_{m+b+k-2}$ существует $i, 1 \leqslant i \leqslant b$, такое, что $h_{m-1+i, 0} \neq h_{m-1+i, 1}$; выберем наименьшее такое $i$.

Чтобы доказать существование у $f=g[h]$ правого барьера длины, меньшей или равной $b+c-1$, надо показать, что ни при каких $x_{1}, \ldots, x_{m+k-2}, y_{m+k}, \ldots, y_{m+b+k-2}$, $z_{m+k}, \ldots, z_{m+b+k-2}$ не выполнена следующая система:

$$
\begin{aligned}
g\left(h_{1,0}, h_{2,0}, \ldots, h_{m, 0}\right) & =g\left(h_{1,1}, h_{2,1}, \ldots, h_{m, 1}\right) \\
g\left(h_{2,0}, h_{3,0}, \ldots, h_{m+1,0}\right) & =g\left(h_{2,1}, h_{3,1}, \ldots, h_{m+1,1}\right) \\
& \ldots \\
g\left(h_{i, 0}, \ldots, h_{m-1+i, 0}\right) & =g\left(h_{i, 1}, \ldots, h_{m-1+i, 1}\right) \\
& \ldots \\
g\left(h_{b+c-1,0}, \ldots, h_{b+c-1+m-1,0}\right) & =g\left(h_{b+c-1,1}, \ldots, h_{b+c-1+m-1,1}\right)
\end{aligned}
$$

Первые $i-1$ уравнений являются тождествами. Рассмотрим $i$-е уравнение системы. Не ограничивая общности, считаем, что

$$
h_{m-1+i, 0}=0, \quad h_{m-1+i, 1}=1 .
$$


По определению индекса $i$, выполнены равенства $h_{i, 0}=h_{i, 1}, \ldots, h_{m-2+i, 0}=h_{m-2+i, 1}$. Отсюда и из определения правого барьера длины $c$ у функции $g$ следует, что система

$$
\begin{aligned}
g\left(h_{i, 0}, h_{i+1,0}, \ldots, h_{m-1+i, 0}\right) & =g\left(h_{i, 1}, h_{i+1,1}, \ldots, h_{m-1+i, 1}\right) \\
g\left(h_{i+1,0}, h_{i+2,0}, \ldots, h_{m+i, 0}\right) & =g\left(h_{i+1,1}, h_{i+2,1}, \ldots, h_{m+i, 1}\right) \\
& \ldots \\
g\left(h_{i+c-1,0}, h_{i+c, 0}, \ldots, h_{i+c-m-2,0}\right) & =g\left(h_{i+c-1,1}, h_{i+c, 1}, \ldots, h_{i+c+m-2,1}\right)
\end{aligned}
$$

не может быть выполнена. Отсюда и из неравенства $i \leqslant b$ получим, что система (11) не выполнена. Это верно для любых $x_{1}, \ldots, x_{m+k-2}, y_{m+k}, \ldots, y_{m+b+k-2}, z_{m+k}, \ldots, z_{m+b+k-2}$, поэтому функция $f=g[h]$ имеет правый барьер длины $d \leqslant b+c-1$.

Неравенство $d \geqslant b$ верно, так как добиться выполнения первых $b-1$ уравнений системы (11) можно, пользуясь лишь разрешимостью системы

$$
\begin{aligned}
h\left(y_{m}, y_{m+1}, \ldots, y_{m+k-1}\right) & =h\left(z_{m}, z_{m+1}, \ldots, z_{m+k-1}\right) \\
h\left(y_{m+1}, y_{m+2}, \ldots, y_{m+k}\right) & =h\left(z_{m+1}, z_{m+2}, \ldots, z_{m+k}\right) \\
& \ldots \\
h\left(y_{m+b-2}, y_{m+b}, \ldots, y_{m+b+k-3}\right) & =h\left(z_{m+b-2}, z_{m+b}, \ldots, z_{m+b+k-3}\right) \\
y_{m}=z_{m}=x_{m}, \ldots, y_{m+k-2}=z_{m+k-2} & =x_{m+k-2}, y_{m+k-1}=0, z_{m+k-1}=1 .
\end{aligned}
$$

Эта система разрешима по определению правого барьера длины $b$ у функции $h$.

Докажем неравенство $d \geqslant c$. Пользуясь наличием у $g$ правого барьера длины $c$, построим пару последовательностей $h_{i, 0}, h_{i+1,0}, \ldots, h_{i+c+m-3,0}, h_{i, 1}, h_{i+1,1}, \ldots, h_{i+c+m-3,1}$, удовлетворяющих системе

$$
\begin{aligned}
g\left(h_{i, 0}, h_{i+1,0}, \ldots, h_{i+m-1,0}\right) & =g\left(h_{i, 1}, h_{i+1,1}, \ldots, h_{i+m-1,1}\right) \\
g\left(h_{i+1,0}, h_{i+2,0}, \ldots, h_{i+m, 0}\right) & =g\left(h_{i+1,1}, h_{i+2,1}, \ldots, h_{i+m, 1}\right) \\
& \ldots \\
g\left(h_{i+c-2,0}, h_{i+c-1,0}, \ldots, h_{i+c+m-3,0}\right) & =g\left(h_{i+c-2,1}, h_{i+c-1,1}, \ldots, h_{i+c+m-3,1}\right) \\
h_{i, 0}=h_{i, 1}, h_{i+1,0} & =h_{i+1,1}, \ldots, h_{i+m-2,0}=h_{i+m-2,1} \\
h_{i+m-1,0} & =h_{i+m-1,1} \oplus 1 .
\end{aligned}
$$

Пользуясь леммой 1 , найдем двоичные последовательности $z, x$ и $y$ такие, что

$$
\begin{aligned}
& h^{*}(x)=\left(z \mid h_{i, 0}, \ldots, h_{i+c+m-3,0}\right), \\
& h^{*}(y)=\left(z \mid h_{i, 1}, \ldots, h_{i+c+m-3,1}\right),
\end{aligned}
$$

и $x_{1}=y_{1}, \ldots, x_{k-1}=y_{k-1}$.

Пусть первый разряд, в котором различаются последовательности $x$ и $y$, - это $q$-й разряд. Положим $i=q-k-m+2$, при этом, если $q-k-m+2 \leqslant 0$, положим $x_{0}=y_{0}=0, x_{-1}=y_{-1}=0, \ldots, x_{q-k-m+2}=y_{q-k-m+2}=0$. Тогда получим, что 
выполнены все равенства следующей системы:

$$
\begin{aligned}
& g\left(h\left(x_{i}, \ldots, x_{i+k-1}\right), h\left(x_{i+1}, \ldots, x_{i+k}\right), \ldots,\right. \\
& \left.h\left(x_{i+m-1}, \ldots, x_{i+k+m-2}\right)\right)=g\left(h\left(y_{i}, \ldots, y_{i+k-1}\right),\right. \\
& h\left(y_{i+1}, \ldots, y_{i+k}\right), \ldots, \\
& \left.h\left(y_{i+m-1}, \ldots, y_{i+k+m-2}\right)\right) \\
& g\left(h\left(x_{i+1}, \ldots, x_{i+k}\right), h\left(x_{i+2}, \ldots, x_{i+k+1}\right), \ldots,\right. \\
& \left.h\left(x_{i+m}, \ldots, x_{i+k+m-1}\right)\right)=g\left(h\left(y_{i+1}, \ldots, y_{i+k}\right),\right. \\
& h\left(y_{i+2}, \ldots, y_{i+k+1}\right), \ldots, \\
& \left.h\left(y_{i+m}, \ldots, y_{i+k+m-1}\right)\right) \\
& g\left(h\left(x_{i+c-2}, \ldots, x_{i+c+k-3}\right),\right. \\
& h\left(x_{i+c-1}, \ldots, x_{i+c+k-2}\right), \ldots, \\
& \left.h\left(x_{i+c+m-3}, \ldots, x_{i+c+k+m-4}\right)\right)=g\left(h\left(y_{i+c-2}, \ldots, y_{i+c+k-3}\right)\right. \text {, } \\
& h\left(y_{i+c-1}, \ldots, y_{i+c+k-2}\right), \ldots, \\
& \left.h\left(y_{i+c+m-3}, \ldots, y_{i+c+k+m-4}\right)\right) \\
& x_{i}=y_{i}, x_{i+1}=y_{i+1}, \ldots, x_{i+k+m-3}=y_{i+k+m-3} \\
& x_{i+k+m-2}=y_{i+k+m-2} \oplus 1 \text {. }
\end{aligned}
$$

По определению, разрешимость системы (13) влечет неравенство $d \geqslant c$, где $d-$ длина правого барьера функции $f$.

Покажем, что каждое из неравенств $d \geqslant \max \{b, c\}$ и $d \leqslant b+c-1$ может как обращаться, так и не обращаться в равенство. Рассмотрим функции

$$
\begin{aligned}
& f_{1}\left(x_{1}, x_{2}, x_{3}, x_{4}\right)=x_{3} \oplus x_{2} x_{4}\left(x_{1} \oplus 1\right), \\
& f_{2}\left(x_{1}, x_{2}, x_{3}, x_{4}\right)=x_{3} \oplus x_{1} x_{4}\left(x_{2} \oplus 1\right)
\end{aligned}
$$

с правыми барьерами длины $b=c=3$. Несложно проверить непосредственно, что у функций $f_{2}\left[f_{2}\right], f_{1}\left[f_{1}\right]$ и $f_{1}\left[f_{2}\right]$ длины правых барьеров равны $d=3, d=4, d=5$ соответственно. Во всех случаях

$$
\max \{b, c\}=3, \quad b+c-1=5,
$$

то есть $d$ принимает все три возможных значения между $\max \{b, c\}$ и $b+c-1$.

Осталось рассмотреть случаи, когда одна или обе из функций $g, h$ не имеют правого барьера. Требуется доказать, что в каждом из этих случаев функция $f$ не имеет правого барьера. Это доказывается в точности теми же рассуждениями, что для неравенств $d \geqslant b$, $d \geqslant c$, с учетом того, что $b$ и (или) $c$ мы можем брать бесконечно большими. Теорема доказана.

В работе [1] описаны все функции с барьером длины 3, аналогичным образом можно описать некоторые классы функций с барьером длины 4. Но эта техника неприменима для описания классов функций без барьеров. А теорема 6 , кроме возможности оценивать длины барьеров функций, порожденных с помощью отображения $\Xi_{m, k}$, также, как мы покажем позже, предоставляет возможность описывать широкие классы функций без барьера. Предварительно докажем два утверждения, позволяющие по известной совершенно уравновешенной функции (с барьерами длины 3 и большей) строить новые совершенно уравновешенные функции со сколь угодно большими длинами барьеров. 
Теорема 7. Пусть $f \in \mathscr{F}_{n}$. Для произвольного положительного цеелого $k$ определим функичию $g_{k} \in \mathscr{F}_{k}(n-1)+1$, полагая

$$
g_{k}\left(x_{1}, \ldots, x_{k(n-1)+1}\right) \equiv f\left(x_{1}, x_{k+1}, x_{2 k+1}, \ldots, x_{k(n-1)+1}\right) .
$$

Тогда

$$
g_{k} \in \mathscr{P P}_{k(n-1)+1} \Longleftrightarrow f \in \mathscr{P P}_{n} .
$$

Доказательство. Пусть $f \in \mathscr{P} \mathscr{P}_{n}$, докажем, что $g_{k} \in \mathscr{P} \mathscr{P}_{k(n-1)+1}$. Предположим противное: пусть $g_{k} \notin \mathscr{P}_{k}(n-1)+1$. Тогда, как следует из теоремы 3 , существуют $r>2 k(n-1)$ и $y, z \in V_{r}$ такие, что выполнена следующая система:

$$
\begin{aligned}
g_{k}\left(y_{1}, \ldots, y_{k(n-1)}, 0\right) & =g_{k}\left(z_{1}, \ldots, z_{k(n-1)}, 1\right) \\
g_{k}\left(y_{2}, \ldots, y_{k(n-1)}, 0, y_{k(n-1)+2}\right) & =g_{k}\left(z_{2}, \ldots, z_{k(n-1)}, 1, z_{k(n-1)+2}\right) \\
\ldots & \\
g_{k}\left(y_{k+1}, \ldots, y_{k(n-1)}, 0, y_{k(n-1)+2}, \ldots, y_{k n+1}\right)= & g_{k}\left(z_{k+1}, \ldots, z_{k(n-1)}, 1,\right. \\
& \left.z_{k(n-1)+2}, \ldots, z_{k n+1}\right) \\
g_{k}\left(y_{k+2}, \ldots, y_{k(n-1)}, 0, y_{k(n-1)+2}, \ldots, y_{k n+2}\right)= & g_{k}\left(z_{k+2}, \ldots, z_{k(n-1)}, 1,\right. \\
& \left.z_{k(n-1)+2}, \ldots, z_{k n+2}\right) \\
\ldots & \\
g_{k}\left(y_{r-k(n-1)}, y_{r-k(n-1)+1}, \ldots, y_{r}\right)= & g_{k}\left(z_{r-k(n-1)}, z_{r-k(n-1)+1}, \ldots, z_{r}\right) \\
y_{1}=z_{1}, y_{2}=z_{2}, \ldots, y_{k(n-1)}=z_{k(n-1)} & \\
y_{r-k(n-1)+1}=z_{r-k(n-1)+1}, y_{r-k(n-1)+2}= & z_{r-k(n-1)+2}, \ldots, y_{r}=z_{r} .
\end{aligned}
$$

Из определения функции $g_{k}$ очевидно, что система (14) эквивалентна системе

$$
\begin{aligned}
f\left(y_{1}, y_{k+1}, y_{2 k+1}, \ldots, y_{k(n-2)+1}, 0\right) & =f\left(z_{1}, z_{k+1}, z_{2 k+1}, \ldots, z_{k(n-2)+1}, 1\right) \\
f\left(y_{2}, y_{k+2}, y_{2 k+2}, \ldots, y_{k(n-2)+2}, y_{k(n-1)+2}\right) & =f\left(z_{2}, z_{k+2}, z_{2 k+2}, \ldots, z_{k(n-2)+2}, z_{k(n-1)+2}\right) \\
& \ldots \\
f\left(y_{k+1}, y_{2 k+1}, \ldots, y_{k(n-2)+1}, 0, y_{k n+1}\right) & =f\left(z_{k+1}, z_{2 k+1}, \ldots, z_{k(n-2)+1}, 1, z_{k n+1}\right) \\
f\left(y_{k+2}, y_{2 k+2}, \ldots, y_{k(n-1)+2}, y_{k n+2}\right) & =f\left(z_{k+2}, z_{2 k+2}, \ldots, z_{k(n-1)+2}, z_{k n+2}\right) \\
& \ldots \\
f\left(y_{l k+1}, y_{(l+1) k+1}, \ldots, y_{(l+n-1) k+1}\right) & =f\left(z_{l k+1}, z_{(l+1) k+1}, \ldots, z_{(l+n-1) k+1}\right) \\
& \ldots \\
f\left(y_{r-k(n-1)}, y_{r-k(n-2)}, \ldots, y_{r}\right) & =f\left(z_{r-k(n-1)}, z_{r-k(n-2)}, \ldots, z_{r}\right) \\
y_{1}=z_{1}, y_{2}=z_{2}, \ldots, y_{k(n-1)}=z_{k(n-1)} & \\
y_{r-k(n-1)+1}=z_{r-k(n-1)+1}, y_{r-k(n-1)+2} & =z_{r-k(n-1)+2}, \ldots, y_{r}=z_{r}
\end{aligned}
$$


Отсюда сразу следует совместность следующей системы:

$$
\begin{gathered}
f\left(y_{1}, y_{k+1} \ldots, y_{k(n-2)+1}, 0\right)=f\left(z_{1}, z_{k+1} \ldots, z_{k(n-2)+1}, 1\right) \\
f\left(y_{k+1}, y_{2 k+1}, \ldots, 0, y_{k n+1}\right)=f\left(z_{k+1}, z_{2 k+1}, \ldots, 0, z_{k n+1}\right) \\
\ldots \\
f\left(y_{l k+1}, y_{(l+1) k+1}, \ldots, y_{(l+n-1) k+1}\right)=f\left(z_{l k+1}, z_{(l+1) k+1}, \ldots, z_{(l+n-1) k+1}\right) \\
y_{1}=z_{1}, y_{k+1}=z_{k+1}, \ldots, y_{k(n-2)+1}=z_{k(n-2)+1} \\
y_{(l+1) k+1}=z_{(l+1) k+1}, y_{(l+2) k+1}=z_{(l+2) k+1}, \ldots, y_{(l+n-1) k+1}=z_{(l+n-1) k+1}
\end{gathered}
$$

Из (16) и из теоремы 3 следует, что $f \notin \mathscr{P}_{n}$, что невозможно.

Обратно, пусть $g_{k} \in \mathscr{P}_{k(n-1)+1}, f \notin \mathscr{P} \mathscr{B}_{n}$. Тогда, по теореме 3 , существует $l \in \mathbf{N}$ такое, что $l+n>2 n-2$, и

$$
\begin{aligned}
& y^{\circ}=\left(y_{1}, y_{k+1}, \ldots, y_{k(n-2)+1}, 0, y_{k n+1}, \ldots, y_{(l+n-1) k+1}\right) \in V_{l+n}, \\
& z^{\circ}=\left(z_{1}, z_{k+1}, \ldots, z_{k(n-2)+1}, 1, z_{k n+1}, \ldots, z_{(l+n-1) k+1}\right) \in V_{l+n},
\end{aligned}
$$

такие, что выполнена система (16). Образуем из $y^{\circ}, z^{\circ}$ пару последовательностей $y, z \in V_{(l+n-1) k+1}$, положив все недостающие компоненты равными нулю. Тогда первое, $(k+1)$-е $, \ldots,(l k+1)$-е уравнения системы (15) выполнены в силу (16), а остальные - в силу равенства $f(0, \ldots, 0)=f(0, \ldots, 0)$. Отсюда видно, что $y, z$ - решение системы (14), следовательно, по теореме $3, g_{k} \notin \mathscr{P P B}_{k(n-1)+1}$. Полученное противоречие завершает доказательство теоремы.

Теорема 8. Функиия $f \in \mathscr{F}_{n}$ имеет правый (левый) барьер длины $b$ тогда и только тогда, когда $g_{k} \in \mathscr{F}_{k(n-1)+1}$ имеет правый (левый) барьер длины $k(b-1)+1$.

Доказательство. Из определения 3 и определения функции $g_{k}$ видно, что если $g_{k}$ имеет правый или левый барьер, то его длина равна $c=k(b-1)+1$ для какого-то натурального $b$. Рассмотрим (для случая правого барьера) $c=k(b-1)+1$ первых уравнений систем (14), (15), именно они определяют наличие правого барьера длины $c$ у функции $g_{k}$. Все уравнения системы (15), кроме 1-го, $(k+1)$-го, $\ldots,(k(b-1)+1)$-го, можно обратить в тривиальные тождества, положив все участвующие в них переменные равными нулю. Останутся $b$ уравнений, определяющих наличие правого барьера длины $b$ у функции $f$, что и доказывает утверждение.

С помощью последнего утверждения можно по известным совершенно уравновешенным функциям с небольшой длиной барьера (если она больше двух) строить совершенно уравновешенные функции со сколь угодно большой длиной барьера. У полученных таким образом функций есть недостаток: расстояние между любыми двумя соседними существенными переменными кратно $k$, что снижает устойчивость фильтрующего генератора с такой функцией к инверсионной атаке Голича (в случае барьера длины 1) и ее аналогу, работающему в случае любого барьера конечной длины. Этот недостаток функции можно легко устранить, воспользовавшись теоремой 5, прибавив к полученной функции $g_{k}$ произвольную функцию из $\mathscr{F} k(n-1)+1$, зависящую только от первых (в случае правого барьера длины $b$ у функции $f) k(n-b)$ переменных.

Таким образом, мы умеем получать функции с большой длиной барьера. Интуитивно понятно, что небольшая длина барьера является слабостью фильтрующей функции. Для случая барьера длины 1 (то есть линейности фильтрующей функции по крайней переменной) Голичем в [6] предложена инверсионная атака на фильтрующий генератор, суть 
которой состоит в возможности эффективно находить прообраз отображения $f^{*}$. Покажем, как можно обобщить эту идею на случай барьера конечной длины у фильтрующей функции. Все рассуждения проведем для случая правого барьера, для левого барьера они совершенно аналогичны.

Теорема 9. Пусть функиия $f \in \mathscr{F}_{n}$ имеет правый барьер длины $b<\infty u$

$$
f^{*}\left(x_{1}, \ldots, x_{m+n-1}\right)=\left(y_{1}, \ldots, y_{m}\right) .
$$

Тогда $\left(x_{1}, \ldots, x_{m+n-b}\right)$ по известным $\left(y_{1}, \ldots, y_{m}\right) u\left(x_{1}, \ldots, x_{n-1}\right)$ можно восстановить со сложностью, не превосходящей $\left(2^{b}-1\right)(m-b+1)$ операций - вычислений значений функции $f$.

Доказательство. Для всех $k$, от $k=n$ и до $k=m+n-b$, повторяем следующую процедуру. Фиксируем произвольным образом следующий неизвестный пока разряд $z_{k}$. Проверяем равенство $f\left(x_{k-n+1}, \ldots, x_{k-1}, z_{k}\right)=y_{k-n+1}$, если оно не выполнено, то $x_{k}=z_{k} \oplus 1$. Если выполнено, проверяем, выполнено ли хотя бы одно из равенств

$$
\begin{aligned}
& f\left(x_{k-n+2}, \ldots, x_{k-1}, z_{k}, 0\right)=y_{k-n+2}, \\
& f\left(x_{k-n+2}, \ldots, x_{k-1}, z_{k}, 1\right)=y_{k-n+2} .
\end{aligned}
$$

Если ни одно из них не выполнено, то очевидно, что $x_{k}=z_{k} \oplus 1$. Иначе, для всех таких $c_{k+1}$, для которых верно равенство $f\left(x_{k-n+2}, \ldots, x_{k-1}, z_{k}, c_{k+1}\right)=y_{k-n+2}$, проверяем равенства

$$
\begin{aligned}
& f\left(x_{k-n+3}, \ldots, x_{k-1}, z_{k}, c_{k+1}, 0\right)=y_{k-n+3}, \\
& f\left(x_{k-n+3}, \ldots, x_{k-1}, z_{k}, c_{k+1}, 1\right)=y_{k-n+3} .
\end{aligned}
$$

Если ни одно из них не выполнено, то $x_{k}=z_{k} \oplus 1$. И так далее, $b$ раз. В итоге, если нашлись такие $c_{k+1}, c_{k+2}, \ldots, c_{k+b-1}$, что

$$
\begin{aligned}
f\left(x_{k-n+1}, \ldots, x_{k-1}, z_{k}\right) & =y_{k-n+1} \\
f\left(x_{k-n+2}, \ldots, x_{k-1}, z_{k}, c_{k+1}\right) & =y_{k-n+2} \\
f\left(x_{k-n+3}, \ldots, x_{k-1}, z_{k}, c_{k+1}, c_{k+2}\right) & =y_{k-n+3} \\
& \cdots \\
f\left(x_{k-n+b}, \ldots, x_{k-1}, z_{k}, c_{k+1}, \ldots, c_{k+b-1}\right) & =y_{k-n+b},
\end{aligned}
$$

то $x_{k}=z_{k}$, в противном случае $x_{k}=z_{k} \oplus 1$, далее увеличиваем $k$ на единицу и переходим к следующему шагу. Здесь мы используем наличие у $f$ правого барьера длины $b$ следующим образом: если $z_{k} \neq x_{k}$, то по определению правого барьера длины $b$ не существует $c_{k+1}, c_{k+2}, \ldots, c_{k+b-1}$, удовлетворяющих системе (17). На протяжении одного шага мы вычисляем значение функции $f$ не более, чем $2^{b}-1$ раз. Суммарная сложность не превосходит $\left(2^{b}-1\right)(m-b+1)$ операций.

Когда $k$ становится больше значения $m+n-b$, оставшиеся $b-1$ переменных $x_{m+n-b+1}, \ldots, x_{m+n-1}$ находятся перебором, что потребует не более $2^{b}-1$ операций вычислений значения функции $f$. Суммарная сложность не превосходит $\left(2^{b}-1\right)(m-b+2)$ операций.

Замечание 2. Оставшиеся $b-1$ неизвестных восстановить однозначно можно не всегда, однако множество удовлетворяющих условию $x_{m+n-b+1}, \ldots, x_{m+n-1}$ легко находится перебором за $2^{b}-2$ операций или меньше. 
Если набор $\left(x_{1}, \ldots, x_{n-1}\right)$ нам не дан, а дана лишь выходная последовательность $\left(y_{1}, \ldots, y_{m}\right)$, то возникает вопрос, с какой вероятностью при случайном выборе набора $\left(x_{1}, \ldots, x_{n-1}\right)$ мы попадем в набор, который является началом какого-то прообраза $\left(y_{1}, \ldots, y_{m}\right)$. Ответ на этот вопрос дает следующая теорема.

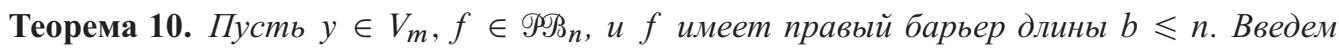
обозначение

$$
X_{y}=f^{*-1}(y)=\left\{x^{1}, \ldots, x^{2^{n-1}}\right\},
$$

для каждого $i, 1 \leqslant i \leqslant 2^{n-1}$, назовем первые $n-1$ разрядов $x^{i}$ начальным состоянием. Тогда число различных начальных состояний в $X_{y}$ не меньше $2^{n-b}$, причем оно равно $2^{n-b}$ тогда и только тогда, когда правый барьер $f$ тривиален, то есть, $f$ не зависит существенно от последнего $b-1$ аргумента и линейна по $(n-b+1)$-му.

Доказательство. Пусть начальные состояния наборов $x^{i}$ и $x^{j}, i \neq j$, равны. Так как $x^{i} \neq x^{j}, x_{1}^{i}=x_{1}^{j}, \ldots, x_{n-1}^{i}=x_{n-1}^{j}$, из определения правого барьера длины $b$ и равенства $f^{*}\left(x^{i}\right)=f^{*}\left(x^{j}\right)$ следует, что $x_{n}^{i}=x_{n}^{j}, \ldots, x_{m+n-b}^{i}=x_{m+n-b}^{j}$, а различаться $x^{i}$ и $x^{j}$ могут только в последних $b-1$ разрядах. Отсюда, для любого начального состояния существует не более $2^{b-1}$ различных соответствующих ему $x^{i}$. Так как $\left|X_{y}\right|=2^{n-1}$, число различных начальных состояний в $X_{y}$ не меньше $2^{n-1} / 2^{b-1}=2^{n-b}$, и у всех $x^{i}$, соответствующих одному начальному состоянию, совпадают первые $m+n-b$ разрядов.

Теперь докажем, что число различных начальных состояний равно $2^{n-b}$ тогда и только тогда, когда правый барьер $f$ тривиален.

Если правый барьер $f$ тривиален, то, так как $f$ не зависит от последних $b-1$ аргументов, $f^{*}\left(x_{1}^{i}, \ldots, x_{m+n-1}^{i}\right)$ однозначно определяется первыми $m+n-b$ разрядами и не зависит от последних $b-1$ разрядов. Отсюда следует, что для любого $y \in V_{m}$ каждому начальному состоянию соответствует не менее $2^{b-1}$ различных $x^{i}$. Поэтому множество $X_{y}$ содержит не более $2^{n-b}$ различных начальных состояний. Из полученного выше результата следует, что $X_{y}$ содержит ровно $2^{n-b}$ различных начальных состояний.

Обратно, пусть для какого-то $y^{\circ} \in V_{m}$ в $X_{y^{\circ}}$ есть ровно $2^{n-b}$ начальных состояний. Возьмем произвольные $x_{m+n}, \ldots, x_{m+2 n-1}$, получим, что

$$
f^{*}\left(x_{1}^{i}, \ldots, x_{m+n-1}^{i}, x_{m+n}, \ldots, x_{m+2 n-1}\right)=\left(y^{\circ} \mid z\right),
$$

причем

$$
\left|f^{*-1}\left(y^{\circ} \mid z\right)\right|=2^{n-1}
$$

так как $f$ совершенно уравновешена. При этом в $f^{*-1}\left(y^{\circ} \mid z\right)$ есть ровно $2^{n-b}$ начальных состояний (меньше быть не может по доказанному выше, больше также не может быть, так как любое начальное состояние $f^{*-1}\left(y^{\circ} \mid z\right)$, очевидно, должно являться и начальным состоянием $\left.f^{*-1}\left(y^{\circ}\right)\right)$. Кроме того, как было показано выше, у всех $x^{i}$, соответствующих одному начальному состоянию $f^{*-1}\left(y^{\circ} \mid z\right)$, обязаны совпадать все разряды, кроме, возможно, последних $b-1$. Учитывая это, получаем, что каждому начальному состоянию $f^{*-1}\left(y^{\circ} \mid z\right)$ соответствует ровно $2^{b-1}$ конечных (состоящих из последних $b-1$ разрядов) наборов битов, откуда следует, что начальному состоянию $\left(x_{1}^{i}, \ldots, x_{n-1}^{i}\right)$ соответствуют наборы $\left(x_{1}^{i}, \ldots, x_{m+n-1}^{i}, x_{m+n}, \ldots, x_{m+2 n-b}, \theta_{1}, \ldots, \theta_{b-1}\right)$, где $\theta_{1}, \ldots, \theta_{b-1}$ принимают все возможные значения. Окончательно получим, что

$$
\begin{aligned}
f\left(x_{m+n}, \ldots, x_{m+2 n-b}, 0, \ldots, 0,0\right) & =f\left(x_{m+n}, \ldots, x_{m+2 n-b}, 0, \ldots, 0,1\right) \\
& =\ldots=f\left(x_{m+n}, \ldots, x_{m+2 n-b}, 1, \ldots, 1,1\right) .
\end{aligned}
$$


Так как значения $x_{m+n}, \ldots, x_{m+2 n-b}$ мы выбирали произвольно, делаем вывод, что $f$ не зависит существенно от последних $b-1$ переменных. Но у $f$, при этом, есть правый барьер длины $b$, а значит, от $(n-b+1)$-й переменной $f$ зависит линейно. Это и требовалось доказать.

Как следствие, получим, что если хотя бы для одного $y \in V_{m}$ верно, что $X_{y}$ имеет ровно $2^{n-b}$ начальных состояний, то это верно и для любого $y \in V_{m}$.

Замечание 3. С помощью теоремы 9 легко строится аналог инверсионной атаки Голича на фильтрующий генератор, эффективный при тех же предположениях, что и инверсионная атака. С увеличением длины барьера эффективность атаки существенно понижается, а в случае фильтрующей функции без барьера этот тип атак не работает вовсе.

Приведем утверждение (доказательство которого можно найти в [7]), с помощью которого мы далее сможем построить широкий класс совершенно уравновешенных функций без барьера.

Лемма 2 ([7]). Функиии вида

$$
\begin{aligned}
f=x_{1} & \oplus x_{m_{1}^{\circ}} x_{m_{1}^{\circ}+1} \ldots x_{m_{1}} h_{1}\left(x_{m_{1}+1}, x_{m_{1}+2}, \ldots, x_{m_{k}}\right) \\
& \oplus x_{m_{2}^{\circ}} x_{m_{2}+1}^{\circ} \ldots x_{m_{2}} h_{2}\left(x_{m_{2}+1}, x_{m_{2}+2}, \ldots, x_{m_{k}}\right) \ldots \\
& \oplus x_{m_{k}} x_{m_{k}+1}^{\circ} \ldots x_{m_{k}}, \quad 1<m_{1}^{\circ}<m_{1}<m_{2}^{\circ}<m_{2}<\ldots<m_{k}^{\circ}<m_{k},
\end{aligned}
$$

где $h_{i}$ - элементарные мономы, а $k$ - нечетное число, являются совершенно уравновешенными функциями без правого барьера.

Теперь с помощью леммы 2, теоремы 8 и теоремы 6 мы можем описать класс совершенно уравновешенных функций без барьера, которому принадлежит, к примеру, функция из примера 1.

Теорема 11. Пусть $f_{1}, f_{2}$ имеют вид (18) или получень из функций вида (18) с помощьюю преобразований

$$
\begin{aligned}
\gamma_{1}: f\left(x_{1}, \ldots, x_{n}\right) & \rightarrow f\left(x_{1} \oplus 1, \ldots, x_{n} \oplus 1\right), \\
f\left(x_{1}, x_{2}, \ldots, x_{n-1}, x_{n}\right) & \rightarrow f\left(x_{1}, x_{k+1}, x_{2 k+1}, \ldots, x_{(n-1) k+1}\right), \quad k \in \mathbf{N} .
\end{aligned}
$$

Тогда функиии вида $f_{1}\left[f_{2}^{\gamma_{2}}\right]$ и $f_{1}^{\gamma_{2}}\left[f_{2}\right]$ являются совершенно уравновешенными функциями без барьера.

Функции, полученные с помощью теоремы 11 , обладают всеми положительными криптографическими качествами совершенно уравновешенных булевых функций, но при этом они также являются устойчивыми против инверсионной атаки Голича и предложенного выше аналога инверсионной атаки, так как у них отсутствует нежелательное для фильтрующей функции свойство барьера конечной длины, которое делает порождаемое функцией отображение $f^{*}$ в некотором смысле обратимым. Это поясняет предпочтительность использования таких функций в качестве фильтрующих.

\section{5. Заключение}

В работе исследованы некоторые свойства булевых функций с барьером конечной длины, выделяющие их из класса всех совершенно уравновешенных функций. Восстановление промежуточного символа входной двоичной последовательности по полученной 
на выходе кодирующего устройства с обладающей правым барьером конечной длины фильтрующей функцией выходной последовательности требует знания только некоторого фиксированного числа последующих выходных символов и предшествующих входных в отличие от случая отсутствия у фильтрующей функции правого барьера, когда в аналогичной ситуации понадобилось бы также знание всей выходной последовательности и части последующих символов входной. Эта принципиальная особенность функций с барьером представляется интересной для дальнейших исследований совершенно уравновешенных функций как порождающих эндоморфизмы динамических систем.

Также одним из интересных направлений дальнейших исследований совершенно уравновешенных булевых функций представляется изучение свойств распределения наборов в прообразах различных выходных последовательностей, связанных с упомянутыми особенностями кодирующих устройств с фильтрующими функциями с барьером.

Кроме того, интересен вопрос о нахождении новых классов функций, обладающих левым барьером, но без правого, с помощью которых изложенным в данной работе методом можно строить широкие классы совершенно уравновешенных функций без барьера.

\section{Список литературы}

1. Логачев О. А., Смышляев С. В., Ященко В. В., Новые методы изучения совершенно уравновешенных булевых функций. Дискретная математика (2009) 21, №2, 51-74.

2. Логачев О. А., Об одном классе совершенно уравновешенных булевых функций. В сб.: Материаль 3-й Международной научной конференции по проблемам безопасности и противодействия терроризму. МЦНМО, Москва, 2008, с. 137-141.

3. Preparata F. P., Convolutional transformations of binary sequences: Boolean functions and their resynchronizing properties. IEEE Trans. Electron. Comput. (1966) 15, №6, 898-909.

4. Сумароков С. Н., Запреты двоичных функций и обратимость для одного класса кодирующих устройств. Обозрение прикладной и промышленной математики (1994) 1, №1, 33-55.

5. Логачев О. А., Сальников А. А., Ященко В. В., Булевы функиии в теории кодирования и криптологии. МЦНМО, Москва, 2004.

6. Golić D. J., On the security of nonlinear filter generators. Lecture Notes Computer Sci. (1996) 1039, 173-188.

7. Смышляев С. В., О некоторых свойствах совершенно уравновешенных булевых функций. В сб.: Материаль 4-й Международной научной конференции по проблемам безопасности и противодействия терроризму. МЦНМО, Москва, 2009, с. 57-64.

Статья поступила 19.08.2009. 